\title{
REALIZATION OF AN ERGODIC MARKOV CHAIN AS A RANDOM WALK SUBJECT TO A SYNCHRONIZING ROAD COLORING
}

\author{
KOUJI YANO, ${ }^{*}$ Kobe University \\ KENJI YASUTOMI,** Ritsumeikan University
}

\begin{abstract}
An ergodic Markov chain is proved to be the realization of a random walk in a directed graph subject to a synchronizing road coloring. The result ensures the existence of appropriate random mappings in Propp-Wilson's coupling from the past. The proof is based on the road coloring theorem. A necessary and sufficient condition for approximate preservation of entropies is also given.
\end{abstract}

Keywords: Markov chain; random walk in a directed graph; road coloring problem; Tsirelson's equation; coupling from the past

2010 Mathematics Subject Classification: Primary 60J10

Secondary 37A35; 05C $81 ; 37 \mathrm{H} 10$

\section{Introduction}

Our aim is to realize an ergodic Markov chain as a suitable random walk in a directed graph, which is generated by a sequence of independent and identically distributed (i.i.d.) random variables taking values in the set of mappings of the state space.

\subsection{Notation}

Let $V$ be a set of finite symbols, say $V=\{1, \ldots, m\}$. Let $Y=\left(Y_{k}\right)_{k \in \mathbb{Z}}$ be a (timehomogeneous) Markov chain taking values in $V$ and indexed by $\mathbb{Z}$, the set of all integers. We write $Q=\left(q_{x, y}\right)_{x, y \in V}$ for the one-step transition probability matrix of $Y$, i.e.

$$
q_{x, y}=\mathrm{P}\left(Y_{1}=y \mid Y_{0}=x\right), \quad x, y \in V .
$$

The $n$th transition probability matrix is given by the $n$th product $Q^{n}=\left(q_{x, y}^{n}\right)_{x, y \in V}$. We call $Y$ irreducible if, for any $x, y \in V$, there exists a positive number $n=n(x, y)$ such that $q_{x, y}^{n}>0$. We call $Y$ aperiodic if the greatest common divisor among $\left\{n \geq 1: q_{x, x}^{n}>0\right\}$ is 1 for all $x \in V$. We call $Y$ ergodic if $Y$ is both irreducible and aperiodic, which is equivalent to the condition that there exists a positive integer $r$ such that $q_{x, y}^{r}>0$ for all $x, y \in V$.

Let $\Sigma$ denote the set of all mappings from $V$ to itself. For $\sigma_{1}, \sigma_{2} \in \Sigma$ and $x \in V$, we simply write $\sigma_{2} \sigma_{1} x$ for $\sigma_{2}\left(\sigma_{1}(x)\right)$.

Received 14 September 2010; revision received 8 March 2011.

* Current address: Graduate School of Science, Kyoto University, Sakyo-ku, Kyoto 606-8502, Japan.

Email address: kyano@math.kyoto-u.ac.jp

Research supported by KAKENHI (20740060).

** Postal address: Department of Mathematical Sciences, Ritsumeikan University, 1-1-1 Noji Higashi, Kusatsu, Shiga 525-8577, Japan. 


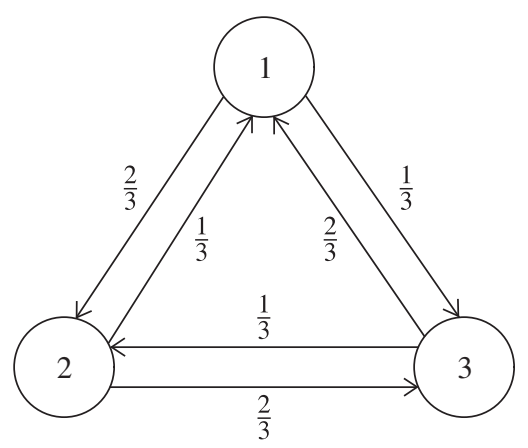

FIGURE 1: Transition probability.

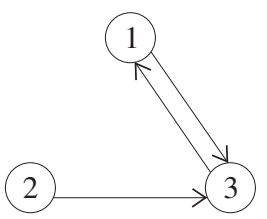

(a)

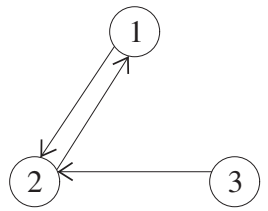

(b)

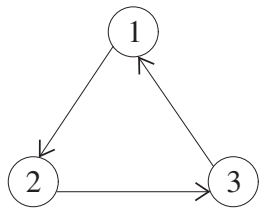

(c)

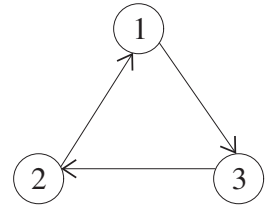

(d)

Figure 2: The elements (a) $\sigma^{(1)}$, (b) $\sigma^{(2)}$, (c) $\sigma^{(3)}$, and (d) $\sigma^{(4)}$ of $\Sigma$.

Definition 1.1. Let $Q$ be the one-step transition probability matrix of a Markov chain. A probability law $\mu$ on $\Sigma$ is called a mapping law for $Q$ if

$$
q_{x, y}=\sum_{\{\sigma \in \Sigma: \sigma x=y\}} \mu(\sigma), \quad x, y \in V .
$$

Definition 1.2. For a probability law $\mu$ on $\Sigma$, a $\mu$-random walk is a Markov chain $(X, N)=$ $\left(X_{k}, N_{k}\right)_{k \in \mathbb{Z}}$ taking values in $V \times \Sigma$ such that $N=\left(N_{k}\right)_{k \in \mathbb{Z}}$ is i.i.d. with common law $\mu$ such that each $N_{k}$ is independent of $\sigma\left(X_{j}, N_{j}: j \leq k-1\right)$ and

$$
X_{k}=N_{k} X_{k-1} \quad \text { almost surely for } k \in \mathbb{Z} .
$$

Let $Y=\left(Y_{k}\right)_{k \in \mathbb{Z}}$ be an ergodic Markov chain with one-step transition probability matrix $Q$. Let $(X, N)$ be a $\mu$-random walk. Then it is obvious that $Y \stackrel{\mathrm{D}}{=} X$ if and only if $\mu$ is a mapping law for $Q$. For any ergodic Markov chain $Y$, we can find a mapping law $\mu$ for $Q$ (see Lemma 3.1).

Let us illustrate our notation. See Figure 1 , where $V=\{1,2,3\}$ and

$$
Q=\left[\begin{array}{lll}
q_{1,1} & q_{1,2} & q_{1,3} \\
q_{2,1} & q_{2,2} & q_{2,3} \\
q_{3,1} & q_{3,2} & q_{3,3}
\end{array}\right]=\left[\begin{array}{ccc}
0 & \frac{2}{3} & \frac{1}{3} \\
\frac{1}{3} & 0 & \frac{2}{3} \\
\frac{2}{3} & \frac{1}{3} & 0
\end{array}\right] .
$$

Let $\sigma^{(1)}, \sigma^{(2)}, \sigma^{(3)}$, and $\sigma^{(4)}$ be elements of $\Sigma$, characterized by Figure 2(a), (b), (c), and (d), respectively. The transition probability $Q$ possesses several mapping laws; among others, we have $\mu^{(1)}$ and $\mu^{(2)}$ defined as

$$
\begin{gathered}
\mu^{(1)}\left(\sigma^{(1)}\right)=\mu^{(1)}\left(\sigma^{(2)}\right)=\mu^{(1)}\left(\sigma^{(3)}\right)=\frac{1}{3}, \\
\mu^{(2)}\left(\sigma^{(3)}\right)=\frac{2}{3}, \quad \mu^{(2)}\left(\sigma^{(4)}\right)=\frac{1}{3} .
\end{gathered}
$$


Identity (1.1) can be checked easily; for instance,

$$
\sum_{\{\sigma \in \Sigma: \sigma(1)=2\}} \mu^{(1)}(\sigma)=\mu^{(1)}\left(\sigma^{(2)}\right)+\mu^{(1)}\left(\sigma^{(3)}\right)=\frac{2}{3}=q_{1,2} .
$$

The two random walks $(X, N)$ corresponding to $\mu^{(1)}$ and $\mu^{(2)}$ have distinct joint laws, but have an identical marginal law of $X$, which is a Markov chain with one-step transition probability $Q$.

\subsection{Realization of an ergodic Markov chain as a $\boldsymbol{\mu}$-random walk}

Our aim is to choose a mapping law $\mu$ which satisfies a nice property.

Definition 1.3. A subset $\Sigma_{0}$ of $\Sigma$ is called synchronizing if there exists a sequence $s=$ $\left(\sigma_{p}, \ldots, \sigma_{1}\right)$ of elements of $\Sigma_{0}$ such that the composition product $\langle s\rangle:=\sigma_{p} \cdots \sigma_{1}$ maps $V$ onto a singleton.

We now introduce one of our main theorems.

Theorem 1.1. Suppose that $Y=\left(Y_{k}\right)_{k \in \mathbb{Z}}$ is ergodic. Then we can choose a mapping law $\mu$ for $Q$ such that $\mu$ has synchronizing support.

Theorem 1.1 will be proved in Section 3.

Let us explain how our $\mu$-random walk is related to road coloring. The support of $\mu$, which we denote by $\left\{\sigma^{(1)}, \ldots, \sigma^{(d)}\right\}$, induces the adjacency matrix $A$ of a directed $\operatorname{graph}(V, A)$ which is of constant outdegree, i.e. from every site there are $d$ roads laid. Then each element $\sigma^{(1)}, \ldots, \sigma^{(d)}$ may be regarded as a road color so that no two roads from the same site have the same color. For a $\mu$-random walk $(X, N)$, the process $X$ moves in the directed graph $(V, A)$ being driven by the randomly chosen road colors indicated by $N$ via (1.2). Thus, we may call $(X, N)$ a random walk in a directed graph subject to a road coloring. For an illustration of the directed graphs induced by $\mu^{(1)}$ and $\mu^{(2)}$, which are defined in (1.3) and (1.4), respectively, see Figure 3(a) and (b), respectively. Since $\sigma^{(1)} \sigma^{(2)} V=\{3\}$, we see that the support of $\mu^{(1)}$ is synchronizing, while we can easily see that the support of $\mu^{(2)}$ is nonsynchronizing.

Let us return to the general discussion. If $(X, N)$ is a $\mu$-random walk and if the support of $\mu$ is synchronizing, then the process $X$ may be represented as

$$
X_{k}=F\left(N_{k}, N_{k-1}, \ldots\right), \quad k \in \mathbb{Z},
$$
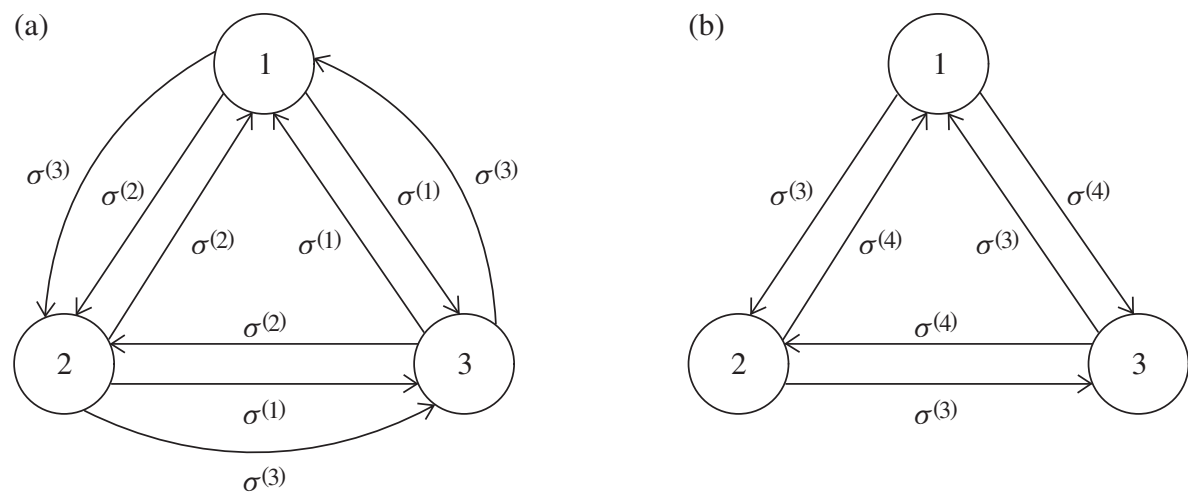

FIGURE 3: The graphs induced by (a) $\mu^{(1)}$ and (b) $\mu^{(2)}$. 
for some measurable function $F: \Sigma^{-\mathbb{N}} \rightarrow V$. In fact, define

$$
T_{k}=\max \left\{l \in \mathbb{Z}, l<k: N_{k} N_{k-1} \cdots N_{l} V \text { is a singleton }\right\},
$$

where we follow the convention that $\max \varnothing=-\infty$. Note that $T_{k}$ is measurable with respect to $\left(N_{j}: j \leq k\right)$. Since the support of $\mu$ is synchronizing, it holds that $T_{k}$ is finite almost surely for all $k \in \mathbb{Z}$, so we may define

$$
X_{k}=N_{k} N_{k-1} \cdots N_{T_{k}} x_{0}, \quad k \in \mathbb{Z},
$$

for a fixed element $x_{0} \in V$, but the resulting random walk does not depend on the choice of $x_{0}$. Such a representation is given in (1.5).

Letting $k=0$ in identity (1.6), we have

$$
X_{0}=N_{0} N_{-1} \cdots N_{T_{0}} x_{0} .
$$

This shows that the stationary law of the Markov chain may be simulated exactly from an i.i.d. sequence. This method was a central idea of Propp-Wilson's coupling from the past (see [15] and also [11, Chapter 10]). Our Theorem 1.1 ensures theoretically that, for any ergodic Markov chain, there always exists an appropriate mapping law such that Propp-Wilson's algorithm terminates almost surely.

For the study of $\mu$-random walks in the case of nonsynchronizing supports, see [19]. Equation (1.2) is called Tsirelson's equation in discrete time; see [3], [12], [13] [20], [21], and [22] for the details.

The representation $Y \stackrel{\mathrm{D}}{=} X=F(N)$ of $Y$ by an i.i.d. sequence $N$ of the form (1.5) is called a nonanticipating representation. Rosenblatt [16], [17] obtained a necessary and sufficient condition for a Markov chain with countable state space to have a nonanticipating representation $Y \stackrel{\mathrm{D}}{=} X=F(N)$, where $N=\left(N_{k}\right)_{k \in \mathbb{Z}}$ is an i.i.d. sequence with uniform law on [0,1].

\subsection{Condition for approximate preservation of entropies}

Let $Y$ and $(X, N)$ be as in Theorem 1.1. We examine the entropy information of $Y$ and $N$. See the standard textbook [4] for basic theory of entropies. Let $\lambda$ be the stationary law of $Y$, and define

$$
h(Y)=-\sum_{x, y \in V} \lambda(x) q_{x, y} \log q_{x, y}
$$

and

$$
h(N)=-\sum_{\sigma \in \Sigma} \mu(\sigma) \log \mu(\sigma) .
$$

Since $Y \stackrel{\mathrm{D}}{=} X$ and $X$ is a measurable function of $N$ as in (1.6), we have

$$
h(Y) \leq h(N) .
$$

Note that Ornstein-Friedman's theorem (see [10] and [14]) asserts that two ergodic Markov chains which have common entropy are isomorphic. By this theorem we see that if the equality holds in (1.7) then $Y$ is isomorphic to $N$. We do not have any general criterion on $Y$ for the existence of a mapping law such that $Y$ is isomorphic to $N$. We will give an example for nonexistence in Section 5.

We are interested in a condition for the existence of mapping laws such that the corresponding $h(N)$ s approximate the $h(Y)$. Following [16], we introduce the following definition. 
Definition 1.4. A Markov chain $Y$ is called $p$-uniform if there exist a probability law $v$ on $V$ and a family $\left\{\tau_{x}: x \in V\right\}$ of permutations of $V$ such that

$$
q_{x, y}=v\left(\tau_{x}(y)\right), \quad x, y \in V .
$$

(The prefix $\mathrm{p}$ is the first letter of 'permutation'.)

Our second main theorem is as follows.

Theorem 1.2. Let $Y$ be an ergodic Markov chain. Then the following assertions are equivalent.

(i) There exists a sequence $\left\{\mu^{(n)}: n=1,2, \ldots\right\}$ of mapping laws for $Q$ with synchronizing support such that the $N^{(n)}$ corresponding to $\mu^{(n)}$ satisfy

$$
h\left(N^{(n)}\right) \rightarrow h(Y) \text { as } n \rightarrow \infty .
$$

(ii) $Y$ is p-uniform.

In particular, if $h(N)=h(Y)$ holds for $N$ corresponding to some mapping law $\mu$ for $Q$ with synchronizing support, then $Y$ is necessarily p-uniform.

Theorem 1.2 will be proved in Section 4.

This paper is organized as follows. In Section 2 we introduce the notation needed to state the road coloring problem. Sections 3 and 4 are devoted to the proofs of Theorems 1.1 and 1.2, respectively. In Section 5 we give an example for Theorem 1.2.

\section{Road colorings of a directed graph}

Let $A=[A(y, x)]_{y, x \in V}$ be a $(V \times V)$-dimensional matrix whose entries are nonnegative integers. The pair $(V, A)$ may be called a directed graph, where, for $x, y \in V$, the value $A(y, x)$ is regarded as the number of directed edges from $x$ to $y$. The set $V$ is called the set of vertices and the matrix $A$ is called the adjacency matrix.

The graph $(V, A)$ is called of constant outdegree if there exists a constant $d$ such that

$$
\sum_{y \in V} A(y, x)=d \quad \text { for all } x \in V .
$$

In this case $(V, A)$ is called $d$-out. The graph $(V, A)$ is called strongly connected if, for any $x, y \in V$, there exists a positive integer $n=n(x, y)$ such that $A^{n}(y, x) \geq 1$. The graph $(V, A)$ is called aperiodic if the greatest common divisor among $\left\{n \geq 1: A^{n}(x, x) \geq 1\right\}$ is 1 for all $x \in V$. Note that $(V, A)$ is both strongly connected and aperiodic if and only if there exists a positive integer $r$ such that $A^{r}(y, x) \geq 1$ for all $x, y \in V$. Following [18], we say that the graph $(V, A)$ or the adjacency matrix $A$ satisfies assumption (AGW) if $(V, A)$ is of constant outdegree, strongly connected, and aperiodic.

Recall that $\Sigma$ is the set of all mappings from $V$ to itself. For $\sigma_{1}, \sigma_{2} \in \Sigma$ and $x \in V$, we simply write $\sigma_{2} \sigma_{1} x$ for $\sigma_{2}\left(\sigma_{1}(x)\right)$. The set $\Sigma$ acts on $V$ in the following sense:

$$
\left(\sigma_{1} \sigma_{2}\right) x=\sigma_{1}\left(\sigma_{2} x\right), \quad \sigma_{1}, \sigma_{2} \in \Sigma, x \in V .
$$

The set $V=\{1, \ldots, m\}$ may be identified with the set of standard basis $\left\{e_{1}, \ldots, e_{m}\right\}$ of $\mathbb{R}^{m}$. An element $\sigma \in \Sigma$ may be identified with the 1-out adjacency matrix $\sigma=[\sigma(y, x)]_{y, x \in V}$ given by

$$
\sigma=\left[\sigma e_{1}, \ldots, \sigma e_{m}\right]
$$


Under these identifications, we see that, for all $x, y \in V$,

$$
\sigma(y, x)=1 \text { if and only if } y=\sigma x .
$$

Let $(V, A)$ be a $d$-out directed graph. A family $\left\{\sigma^{(1)}, \ldots, \sigma^{(d)}\right\}$ of elements of $\Sigma$ is called a road coloring of $(V, A)$ if the following identity holds:

$$
A=\sigma^{(1)}+\cdots+\sigma^{(d)} .
$$

Every road is assigned a color chosen from the $d$ colors $\left\{\sigma^{(1)}, \ldots, \sigma^{(d)}\right\}$. Here we remark that the elements $\sigma^{(1)}, \ldots, \sigma^{(d)}$ are not necessarily distinct. For an illustration, consider

$$
A=\left[\begin{array}{lll}
0 & 2 & 1 \\
1 & 0 & 2 \\
2 & 1 & 0
\end{array}\right]
$$

and see Figure 3(a) in Section 1. In this case we have $A=\sigma^{(1)}+\sigma^{(2)}+\sigma^{(3)}$ and, hence, $\left\{\sigma^{(1)}, \sigma^{(2)}, \sigma^{(3)}\right\}$ is a road coloring of $(V, A)$. Here we remark that the family $\left\{\sigma^{(3)}, \sigma^{(3)}, \sigma^{(4)}\right\}$ is another road coloring of $(V, A)$ which is different from $\left\{\sigma^{(1)}, \sigma^{(2)}, \sigma^{(3)}\right\}$.

Note that, for any graph $(V, A)$ of constant outdegree, there exists at least one road coloring of $(V, A)$. Conversely, if we are given a family $\left\{\sigma^{(1)}, \ldots, \sigma^{(d)}\right\}$ of elements of $\Sigma$, then it induces a unique $d$-out directed graph $(V, A)$, where $A$ is defined by (2.1).

Let $\Sigma_{0}$ be a subset of $\Sigma$. A sequence $s=\left(\sigma_{p}, \ldots, \sigma_{2}, \sigma_{1}\right)$ of elements of $\Sigma_{0}$ is called a $\Sigma_{0}$-word. For a $\Sigma_{0}$-word $s=\left(\sigma_{p}, \ldots, \sigma_{2}, \sigma_{1}\right)$, we write $\langle s\rangle$ for the product $\sigma_{p} \cdots \sigma_{2} \sigma_{1}$. The following definition is a slight modification of Definition 1.3.

Definition 2.1. A road coloring $\Sigma_{0}=\left\{\sigma^{(1)}, \ldots, \sigma^{(d)}\right\}$ is called synchronizing if $\Sigma_{0}$ as a subset of $\Sigma$ is synchronizing.

By this definition we see that a road coloring $\Sigma_{0}=\left\{\sigma^{(1)}, \ldots, \sigma^{(d)}\right\}$ is synchronizing if and only if $\langle s\rangle V$ is a singleton for some $\Sigma_{0}$-word $s$. If we express

$$
s=\left(\sigma^{(i(p))}, \ldots, \sigma^{(i(2))}, \sigma^{(i(1))}\right)
$$

for some numbers $i(1), \ldots, i(p) \in\{1, \ldots, d\}$, the assertion ' $\langle s\rangle V$ is a singleton' may be stated in other words as follows. Those who walk in the graph $(V, A)$ according to the colors $\sigma^{(i(1))}, \ldots, \sigma^{(i(p))}$ in this order will lead to a common vertex, no matter where they started from.

Now we state the road coloring theorem.

Theorem 2.1. ([18].) Suppose that the directed graph $(V, A)$ satisfies assumption $(A G W)$. Then there exists a synchronizing road coloring of $(V, A)$.

This was first conjectured in the case of no multiple directed edges by Adler et al. [1] (see also [2, Section 11]) in the context of the isomorphism problem of symbolic dynamics with common topological entropy. For related studies published prior to that of Trahtman [18], see [8] and [9]; see also [5], [6], and [7].

\section{Construction of a mapping law on a synchronizing road coloring}

We need the following lemma.

Lemma 3.1. Let $Y$ be a Markov chain with one-step transition probability matrix $Q$. Then there exists a mapping law $\mu$ for $Q$. 
Proof. First, we suppose that $q_{x, y}$ is a rational number for all $x, y \in V$. Then we may take an integer $d$ sufficiently large so that $A(y, x):=q_{x, y} d$ is an integer for all $x, y \in V$. Then $A:=[A(y, x)]_{x, y \in V}$ is the adjacency matrix of a $d$-out directed graph $(V, A)$; in fact,

$$
\sum_{y \in V} A(y, x)=d \sum_{y \in V} q_{x, y}=d .
$$

Let $\left\{\sigma^{(1)}, \ldots, \sigma^{(d)}\right\}$ be a road coloring of $(V, A)$, and define

$$
\mu(\sigma)=\frac{1}{d} \#\left(\left\{i=1, \ldots, d: \sigma^{(i)}=\sigma\right\}\right),
$$

where \#(·) denotes the number of elements of the set indicated. Thus, for any $x, y \in V$, we see that

$$
\sum_{\{\sigma \in \Sigma: y=\sigma x\}} \mu(\sigma)=\frac{1}{d} \#\left(\left\{i=1, \ldots, d: \sigma^{(i)}(y, x)=1\right\}\right)=\frac{1}{d} A(y, x)=q_{x, y},
$$

which shows that $\mu$ is a mapping law for $Q$.

Second, we consider the general case. Let us take a sequence $\left\{Q^{(n)}: n=1,2, \ldots\right\}$ of onestep transition probability matrices such that $q_{x, y}^{(n)}$ is a rational number for all $n$ and $x, y \in V$ and that $q_{x, y}^{(n)} \rightarrow q_{x, y}$ as $n \rightarrow \infty$ for all $x, y \in V$. Then, for any $n$, there exists a mapping law $\mu^{(n)}$ for $Q^{(n)}$. Since $\Sigma$ is a finite set, we can choose some subsequence $\left\{\mu^{(n(k))}: k=1,2, \ldots\right\}$ and some probability law $\mu$ on $\Sigma$ such that $\mu^{(n(k))}(\sigma) \rightarrow \mu(\sigma)$ as $k \rightarrow \infty$. This shows that $\mu$ is a mapping law for $Q$. This completes the proof.

Now we proceed to prove Theorem 1.1.

Proof of Theorem 1.1. Let $Q=\left(q_{x, y}\right)_{x, y \in V}$ be the one-step transition probability matrix for an ergodic Markov chain $Y$.

First, we take an adjacency matrix $A$ which is of constant outdegree and satisfies

$$
A(y, x) \begin{cases}\geq 1 & \text { if } q_{x, y}>0 \\ =0 & \text { if } q_{x, y}=0\end{cases}
$$

For this, we introduce a subset $V \times V$ defined by

$$
E=\left\{(x, y) \in V \times V: q_{x, y}>0\right\} .
$$

For each $x \in V$, we define the outdegree of $E$ at $x$ by

$$
d(x)=\#\{(x, y) \in E: y \in V\},
$$

and write $d=\max _{x \in V} d(x)$ for the maximum outdegree of $E$. For each $x \in V$, we choose a site $\sigma(x) \in V$ so that $(x, \sigma(x)) \in E$. Now we set

$$
A(y, x)= \begin{cases}d-d(x)+1 & \text { if } y=\sigma(x), \\ 1 & \text { if } y \neq \sigma(x) \text { and }(x, y) \in E, \\ 0 & \text { otherwise. }\end{cases}
$$

Then this $(A(y, x))_{x, y \in V}$ is of constant outdegree and satisfies (3.1). 
Since $Y$ is an ergodic Markov chain, there exists a positive integer $r$ such that $q_{x, y}^{r}>0$ for all $x, y \in V$. Hence, we have $A^{r}(y, x) \geq 1$ for all $x, y \in V$; in fact, there exists a path $x=x_{0}, x_{1}, \ldots, x_{n}=y$ such that $q_{x_{k-1}, x_{k}}>0$ for $k=1,2, \ldots, n$, which implies that $A\left(x_{k}, x_{k-1}\right) \geq 1$ for $k=1,2, \ldots, n$. Thus, we see that $(V, A)$ satisfies assumption (AGW). This means that we can apply Theorem 2.1 to obtain a synchronizing road coloring $\left\{\sigma^{(1)}, \ldots, \sigma^{(d)}\right\}$ of $(V, A)$. Define

$$
\hat{\mu}(\sigma)=\frac{1}{d} \#\left(\left\{i=1, \ldots, d: \sigma^{(i)}=\sigma\right\}\right), \quad \sigma \in \Sigma,
$$

and define

$$
\hat{q}_{x, y}=\sum_{\{\sigma \in \Sigma: y=\sigma x\}} \hat{\mu}(\sigma), \quad x, y \in V .
$$

Then $\hat{\mu}$ is a mapping law for $\hat{Q}$ and has synchronizing support. We also note that

$$
\hat{q}_{x, y}=0 \quad \text { if }(x, y) \notin E .
$$

Let

$$
\varepsilon=\min \left\{q_{x, y}:(x, y) \in E\right\}>0 .
$$

If $\varepsilon=1$ then we have $Q=\hat{Q}$, so that $\hat{\mu}$ is as desired. Let us assume that $\varepsilon<1$. Define

$$
Q^{(\varepsilon)}=\frac{1}{1-\varepsilon}(Q-\varepsilon \hat{Q})
$$

Then $Q^{(\varepsilon)}=\left(q_{x, y}^{(\varepsilon)}\right)_{x, y \in V}$ is a one-step transition probability matrix of a Markov chain. In fact, we see that

$$
(1-\varepsilon) q_{x, y}^{(\varepsilon)}=q_{x, y}-\varepsilon \hat{q}_{x, y} \geq q_{x, y}-\varepsilon \mathbf{1}_{\{(x, y) \in E\}} \geq 0, \quad x, y \in V,
$$

and that

$$
\sum_{y \in V} q_{x, y}^{(\varepsilon)}=\frac{1}{1-\varepsilon}\left(\sum_{y \in V} q_{x, y}-\varepsilon \sum_{y \in V} \hat{q}_{x, y}\right)=1 .
$$

Now we apply Lemma 3.1 to obtain a mapping law $\mu^{(\varepsilon)}$ for $Q^{(\varepsilon)}$. Define

$$
\mu=(1-\varepsilon) \mu^{(\varepsilon)}+\varepsilon \hat{\mu} .
$$

Since $\mu^{(\varepsilon)}$ has synchronizing support, so does $\mu$. For $x, y \in V$, we have

$$
\begin{aligned}
\sum_{\{\sigma \in \Sigma: y=\sigma x\}} \mu(\sigma) & =(1-\varepsilon) \sum_{\{\sigma \in \Sigma: y=\sigma x\}} \mu^{(\varepsilon)}(\sigma)+\varepsilon \sum_{\{\sigma \in \Sigma: y=\sigma x\}} \hat{\mu}(\sigma) . \\
& =(1-\varepsilon) q_{x, y}^{(\varepsilon)}+\varepsilon \hat{q}_{x, y} \\
& =q_{x, y}
\end{aligned}
$$

which shows that $\mu$ is a mapping law for $Q$. This completes the proof. 


\section{Approximate preservation of entropies}

Let us prove Theorem 1.2.

Proof of Theorem 1.2. We prove that (i) implies (ii). Note that

$$
\begin{aligned}
h(Y) & =-\sum_{x, y \in V} \lambda(x) q_{x, y} \log q_{x, y}, \\
h\left(N^{(n)}\right) & =-\sum_{\sigma \in \Sigma} \mu^{(n)}(\sigma) \log \mu^{(n)}(\sigma) .
\end{aligned}
$$

Taking a subsequence if necessary, we assume that there exists a probability law $\mu$ on $\Sigma$ such that $\mu^{(n)}(\sigma) \rightarrow \mu(\sigma)$ for all $\sigma \in \Sigma$. Note that $\mu$ is a mapping law for $Q$ but does not necessarily have synchronizing support. By assumption (1.9) we see that

$$
h(Y)=\lim _{n \rightarrow \infty} h\left(N^{(n)}\right)=-\sum_{\sigma \in \Sigma} \mu(\sigma) \log \mu(\sigma) .
$$

For $x, y \in V$, we set

$$
\Sigma(y, x)=\{\sigma \in \Sigma: y=\sigma x\},
$$

so that we have

$$
q_{x, y}=\sum_{\sigma \in \Sigma(y, x)} \mu(\sigma)
$$

Hence, we have

$$
\mu(\sigma) \leq q_{x, y} \quad \text { whenever } \sigma \in \Sigma(y, x) .
$$

Since $t \mapsto \log t$ is increasing, we have

$$
-\sum_{\sigma \in \Sigma(y, x)} \mu(\sigma) \log \mu(\sigma) \geq-\sum_{\sigma \in \Sigma(y, x)} \mu(\sigma) \log q_{x, y}=-q_{x, y} \log q_{x, y} .
$$

Since $\bigcup_{y \in V} \Sigma(y, x)=\Sigma$, we have

$$
h(Y)=-\sum_{y \in V} \sum_{\sigma \in \Sigma(y, x)} \mu(\sigma) \log \mu(\sigma) \geq q(x) \text { for all } x \in V,
$$

where we set

$$
q(x)=-\sum_{y \in V} q_{x, y} \log q_{x, y}, \quad x \in V .
$$

We take $\hat{x} \in V$ such that

$$
q(\hat{x})=\max _{x \in V} q(x) .
$$

Using (4.4) and (4.1), we have

$$
q(\hat{x}) \leq h(Y)=\sum_{x \in V} \lambda(x) q(x) \leq q(\hat{x}) .
$$

Thus, we see that the equalities hold in (4.5) and that $q(x)=q(\hat{x})$ for all $x \in V$. For any $x \in V$, we combine $h(N)=q(x)$ together with (4.3) to obtain

$$
-\sum_{\sigma \in \Sigma(y, x)} \mu(\sigma) \log \mu(\sigma)=-q_{x, y} \log q_{x, y}, \quad x, y \in V .
$$


Combining this with (4.2), we obtain

$$
\mu(\sigma)=q_{x, y} \quad \text { whenever } \sigma \in \Sigma(y, x) .
$$

Let $x_{0} \in V$ be fixed, and let $x \in V$. Since $\{\Sigma(y, x): y \in V\}$ is a partition of $\Sigma$, we may choose a permutation $\tau_{x}$ of $V$ so that

$$
\Sigma\left(\tau_{x}(y), x\right) \cap \Sigma\left(y, x_{0}\right) \neq \varnothing, \quad y \in V .
$$

This shows that

$$
q_{x, \tau_{x}(y)}=q_{x_{0}, y}, \quad x, y \in V,
$$

which implies p-uniformity of $Y$. This completes the proof of the implication (i) $\Rightarrow$ (ii).

We now prove that (ii) implies (i). Let $\left\{x_{1}, \ldots, x_{d}\right\}$ be an enumeration of the support of the law $v$ in (1.8). For $i=1, \ldots, d$, we define

$$
\sigma^{(i)}(y, x)=\mathbf{1}_{\left\{\tau_{x}(y)=x_{i}\right\}} .
$$

For each $x \in V$, there exists a unique $y \in V$ such that $\sigma^{(i)}(y, x)=1$, so that we have $\sigma^{(i)} \in \Sigma$. By (1.8) we obtain

$$
q_{x, y}=\sum_{i=1}^{d} \sigma^{(i)}(y, x) v\left(x_{i}\right), \quad x, y \in V .
$$

Let $A$ be as in (3.1), and let $\Sigma_{1}$ be a synchronizing subset corresponding to some synchronizing road coloring of $(V, A)$. For a sufficiently large integer $n$, we define a probability law $\mu^{(n)}$ on $\Sigma$ by

$$
\mu^{(n)}(\sigma)=\sum_{\left\{i: \sigma^{(i)}=\sigma\right\}}\left\{v\left(x_{i}\right)-\frac{1}{n d}\right\}+\frac{1}{n\left|\Sigma_{1}\right|} \mathbf{1}_{\left\{\sigma \in \Sigma_{1}\right\}} .
$$

Then it is obvious that $\mu^{(n)}$ is a mapping law for $Q$ and has synchronizing support.

Let us verify condition (1.9). On the one hand, we have

$$
h\left(N^{(n)}\right) \rightarrow-\sum_{i=1}^{d} v\left(x_{i}\right) \log v\left(x_{i}\right) \quad \text { as } n \rightarrow \infty .
$$

On the other hand, we have

$$
\begin{aligned}
h(Y) & =-\sum_{x, y \in V} \lambda(x) q_{x, y} \log q_{x, y} \\
& =-\sum_{x, y \in V} \lambda(x) \sum_{i=1}^{d} \sigma^{(i)}(y, x) v\left(x_{i}\right) \log v\left(x_{i}\right) \\
& =-\sum_{i=1}^{d}\left\{\sum_{x, y \in V} \lambda(x) \sigma^{(i)}(y, x)\right\} v\left(x_{i}\right) \log v\left(x_{i}\right) \\
& =-\sum_{i=1}^{d} v\left(x_{i}\right) \log v\left(x_{i}\right) .
\end{aligned}
$$

This shows (1.9), completing the proof. 


\section{An example}

Let $V=\{1,2\}$. Then $\Sigma=\{(12),(21),(11),(22)\}$, where

$$
(i j)=\left[\begin{array}{c}
1 \mapsto i \\
2 \mapsto j
\end{array}\right], \quad i, j=1,2 .
$$

Let $0<p<1$, and consider a Markov chain $Y$ with one-step transition probability given by

$$
\left[\begin{array}{ll}
q_{1,1} & q_{1,2} \\
q_{2,1} & q_{2,2}
\end{array}\right]=\left[\begin{array}{cc}
p & 1-p \\
1-p & p
\end{array}\right] .
$$

Then it is obvious that $Y$ is an ergodic Markov chain. Since

$$
\left[\begin{array}{l}
q_{1,1} \\
q_{2,1}
\end{array}\right]=\left[\begin{array}{l}
q_{2,2} \\
q_{1,2}
\end{array}\right]=\left[\begin{array}{c}
p \\
1-p
\end{array}\right],
$$

we see that $Y$ is p-uniform.

It is obvious that the stationary law is given as

$$
\lambda(1)=\lambda(2)=\frac{1}{2}
$$

We now see that

$$
h(Y)=\varphi(p)+\varphi(1-p)
$$

where $\varphi(t)=-t \log t$.

If $\mu$ is a mapping law for $Q$ then we have

$$
\mu(12)+\mu(11)=p, \quad \mu(21)+\mu(11)=1-p .
$$

From this, we see that there exists some $\varepsilon$ with $0 \leq \varepsilon \leq \min \{p, 1-p\}$ such that

$$
\varepsilon=\mu(11)=\mu(22), \quad \mu(12)=p-\varepsilon, \quad \mu(21)=1-p-\varepsilon .
$$

Conversely, for any $\varepsilon$ with $0 \leq \varepsilon \leq \min \{p, 1-p\}$, we may define $\mu=\mu^{(\varepsilon)}$ by (5.1) so that $\mu^{(\varepsilon)}$ is a mapping law for $Q$.

If $\mu^{(\varepsilon)}$ has synchronizing support, $\varepsilon$ should be positive. Let $\left\{X^{(\varepsilon)}, N^{(\varepsilon)}\right\}$ be the $\mu^{(\varepsilon)}$-random walk. We then see that

$$
h\left(N^{(\varepsilon)}\right)=2 \varphi(\varepsilon)+\varphi(p-\varepsilon)+\varphi(1-p-\varepsilon) .
$$

If $p=\frac{1}{2}$, we see that $h(Y)=h\left(N^{(1 / 2)}\right)$.

Suppose that $p \neq \frac{1}{2}$. Then, by an easy computation we see that

$$
h(Y)<h\left(N^{(\varepsilon)}\right)
$$

for all $\varepsilon$ with $0<\varepsilon \leq \min \{p, 1-p\}$. However, it holds that $h\left(N^{(\varepsilon)}\right) \rightarrow h(Y)$ as $\varepsilon \rightarrow 0+$. 


\section{References}

[1] Adler, R. L., Goodwyn, L. W. AND Weiss, B. (1977). Equivalence of topological Markov shifts. Israel J. Math., 27, 48-63.

[2] Adler, R. L. And Weiss, B. (1970). Similarity of Automorphisms of the Torus (Memoirs Amer. Math. Soc. 98). American Mathematical Society, Providence, RI.

[3] Akahori, J., Uenishi, C. and Yano, K. (2008). Stochastic equations on compact groups in discrete negative time. Prob. Theory Relat. Fields 140, 569-593.

[4] Billingsley, P. (1978). Ergodic Theory and Information. Robert E. Krieger Publishing, Huntington, NY.

[5] Budzban, G. (2004). Semigroups and the generalized road coloring problem. Semigroup Forum 69, 201-208.

[6] Budzban, G. And Feinsilver, P. (2007). Completely simple semigroups, Lie algebras, and the road coloring problem. Semigroup Forum 74, 206-226.

[7] Budzban, G. And MukherJea, A. (2000). A semigroup approach to the road coloring problem. In Probability on Algebraic Structures (Gainesville, FL, 1999; Contemp. Math. 261), American Mathematical Society, Providence, RI, pp. 195-207.

[8] Culik, K., II, KarhumäKI, J. And KarI, J. (2002). A note on synchronized automata and road coloring problem. In Developments in Language Theory (Vienna, 2001; Lecture Notes Comput. Sci. 2295), Springer, Berlin, pp. 175-185.

[9] Friedman, J. (1990). On the road coloring problem. Proc. Amer. Math. Soc. 110, 1133-1135.

[10] Friedman, N. A. And Ornstein, D. S. (1970). On isomorphism of weak Bernoulli transformations. Adv. Math. 5, 365-394.

[11] HäGgström, O. (2002). Finite Markov Chains and Algorithmic Applications (London Math. Soc. Student Texts 52). Cambridge University Press.

[12] Hirayama, T. and Yano, K. (2010). Extremal solutions for stochastic equations indexed by negative integers and taking values in compact groups. Stoch. Process. Appl. 120, 1404-1423.

[13] Hirayama, T. ANd Yano, K. (2010). Strong solutions of Tsirelson's equation in discrete time taking values in compact spaces with semigroup action. Preprint. Available at http://arxiv.org/abs/1005.0038v1.

[14] Ornstein, D. (1970). Bernoulli shifts with the same entropy are isomorphic. Adv. Math. 4, 337-352.

[15] Propp, J. G. and Wilson, D. B. (1996). Exact sampling with coupled Markov chains and applications to statistical mechanics. Internat. Random Structures Algorithms 9, 223-252.

[16] Rosenblatt, M. (1959). Stationary processes as shifts of functions of independent random variables. J. Math. Mech. 8, 665-681.

[17] Rosenblatt, M. (1962). Stationary Markov chains and independent random variables. J. Math. Mech. 9, 945949. (Addendum: 11 (1962), 317.)

[18] Trahtman, A. N. (2009). The road coloring problem. Israel J. Math. 172, 51-60.

[19] YANo, K. (2010). Random walk in a finite directed graph subject to a road coloring. Preprint. Available at http://arxiv.org/abs/1005.0079v3.

[20] Yano, K. And TAKahashi, Y. (2007). Time evolution with and without remote past. Sūrikaisekikenkyūsho Kōkyūroku 1552, 164-171.

[21] YANO, K. AND Yor, M. (2010). Around Tsirelson's equation, or: the evolution process may not explain everything. Preprint. Available at http://arxiv.org/abs/0906.3442v2.

[22] Yor, M. (1992). Tsirel'son's equation in discrete time. Probab. Theory Relat. Fields 91, 135-152. 